\title{
Magnetic-field Induced Screening Effect and Collective Excitations
}

\author{
Kenji Fukushima \\ Department of Physics, Keio University, Kanagawa 223-8522, Japan
}

\begin{abstract}
We explicitly construct the fermion propagator in a magnetic field background $B$ to take the lowest Landau-level approximation. We analyze the energy and momentum dependence in the polarization tensor and discuss the collective excitations. We find there appear two branches of collective modes in one of two transverse gauge particles; one represents a massive and attenuated gauge particle and the other behaves similar to the zero sound at finite density.

PACS numbers: 12.38.Mh, 25.75.Ag, 12.38.-t, 12.20.-m
\end{abstract}

Systems in the extreme environment with intense fields 1] are attracting theoretical interest recently because experimental developments are making it a real possibility to observe exotic phenomena in strong fields. In quantum electrodynamics (QED) a pair production is expected under a strong electric field $E$, which is called the Schwinger mechanism, and the critical strength of $E$ necessary for the electron-positron production is given by an order of $|e E| \sim m_{e}^{2}$, i.e. $E \sim m_{e}^{2} / e \sim 10^{16} \mathrm{~V} / \mathrm{cm}$. Such a strong field is still beyond the experimentally reachable limit. It is known that optimization of spatial and temporal profiles of the pulse laser could significantly lower the critical $E$, which enhances a chance further to realize the Schwinger pair production in the laboratory 2].

A magnetic field $B$ is also of paramount importance. Of course a time-dependent $E$ would be generally accompanied by $B$. Moreover, the effect caused by $B$ itself is interesting enough; one well-known example is a phenomenon called the magnetic catalysis [3] which favors a $B$-induced chiral condensate. The birefringence in a medium of strong $B$ can also be a sizable effect for $|e B| \sim m_{e}^{2}$, which requires $B \sim m_{e}^{2} / e \sim 10^{13}$ gauss. This is again far beyond the limit of experimentally obtainable intensity, while this critical $B$ could be of order of the surface strength on the neutron star or magnetar in nature.

One might have thought that it is difficult to attain such large $B$ that any new phenomenon can be realized in the laboratory. A new possibility was then brought from physics of the relativistic heavy-ion collision experiment which aims to create a new state of matter out of quarks and gluons (called the quark-gluon plasma). The heavy-ion collision experiments have turned out to be successful particularly at RHIC in BNL and more data is coming from LHC in CERN. Because heavy ions are positively charged and move at almost the speed of light, they can generate a gigantic magnetic field if their collision geometry is non-central. It was shown that the maximum $B$ reached at RHIC energy is as large as $|e B| \sim m_{\pi}^{2} \sim 10^{18}$ gauss [4, 5]. This is no longer a QED energy scale but should be already relevant to physics of quantum chromodynamics (QCD) such as the chiral magnetic effect, the chiral vortical effect, the chiral separation effect, and so on [4, 6].

Physical properties of QED and QCD matter in an in- tense $B$ field are under active investigations by means of analytical and numerical studies. In the Monte-Carlo simulation an introduction of strong $B$ is feasible and various observables have been measured in the latticeQCD calculation [7] as well as model studies on the QCD phase transitions [8] and on the color superconducting state of dense quark matter [9]. In this work we will explore the $B$-induced screening effect in an analytical approach, which is analogous to the screening in the finitetemperature field theory.

First of all let us write down the fermion propagator. This part might look technical but explicit calculations are useful to clarify how the momentum conservation holds. Using Ritus' method [10] we can explicitly solve the free Dirac equation. In the following we use Landau's gauge fixing, i.e. $A^{0}=A^{1}=A^{3}=0$ and $A^{2}=B x$, which describes $B$ in the third direction. We here assume $e B>0$, though we can easily relax this with minor modification. We define the projection matrix [9] as $P_{k}(x)=\operatorname{diag}\left[f_{k+}(x), f_{k-}(x), f_{k+}(x), f_{k-}(x)\right]$ with the Landau wave-functions;

$$
\begin{aligned}
& f_{k+}(x)=\phi_{k}\left(x^{1}-p^{2} / e B\right) \quad(k=0,1, \ldots) \\
& f_{k-}(x)=\phi_{k-1}\left(x^{1}-p^{2} / e B\right) \quad(k=1,2, \ldots) .
\end{aligned}
$$

These are the expressions for $e B>0$ and $f_{k+}$ and $f_{k-}$ should be swapped if $e B<0$. Here $\phi_{k}(x)$ is the wavefunction for the harmonic oscillator with $k$ quanta.

One can then prove that the background of $A^{2}=B x$ is eliminated with this projection matrix and the momentum is replaced accordingly as $\boldsymbol{p} \rightarrow \tilde{\boldsymbol{p}}=\left(0,-\sqrt{2 e B k}, p^{3}\right)$. Now that we have the complete basis of the solutions of the Dirac equation it is straightforward to write down the two-point functions in a standard procedure;

$$
\begin{aligned}
& \langle\psi(x) \bar{\psi}(y)\rangle \\
& =\int \frac{\mathrm{d} p^{2} \mathrm{~d} p^{3}}{(2 \pi)^{2}} \sum_{k} \frac{\mathrm{e}^{-\mathrm{i} \omega_{p}\left(x^{0}-y^{0}\right)+\mathrm{i} p^{2}\left(x^{2}-y^{2}\right)+\mathrm{i} p^{3}\left(x^{3}-y^{3}\right)}}{2 \omega_{p}} \\
& \quad \times P_{k}(x)\left(\omega_{p} \gamma^{0}-\tilde{\boldsymbol{p}} \cdot \boldsymbol{\gamma}+m\right) P_{k}(y),
\end{aligned}
$$

which represents the particle propagation from $y$ to $x$. We note that $\omega_{p}=\sqrt{\tilde{\boldsymbol{p}}^{2}+m^{2}}$ is the particle energy. In the same way we get $\langle\bar{\psi}(y) \psi(x)\rangle$ for the anti-particle propagation from $x$ to $y$, from which we can get the retarded, advanced, and Feynman propagators. 


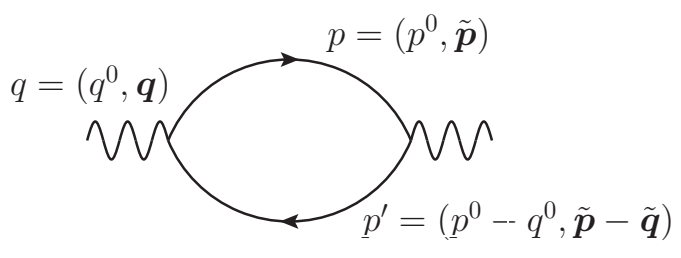

FIG. 1. One-loop diagram of the polarization tensor. The external momentum $q$ is not Landau quantized, while the momenta in the loop are quantized and replaced by $\tilde{\boldsymbol{p}}$ and $\tilde{\boldsymbol{q}}$, which are further reduced to $\overline{\boldsymbol{p}}$ and $\overline{\boldsymbol{q}}$ in the LLL approximation.

We cannot, however, express those propagators in a compact form using the i $\epsilon$-prescription because $P_{k}$ is not commutative with $\gamma^{2}$. In the lowest Landau-level (LLL) approximation that we adopt hereafter, the term involving $\gamma^{2}$ is dropped and the Feynman propagator for example simplifies into

$$
\begin{aligned}
& S_{0}^{F}(x, y)=\langle T \psi(x) \bar{\psi}(y)\rangle_{0} \\
& =\int \frac{\mathrm{d} p^{0} \mathrm{~d} p^{2} \mathrm{~d} p^{3}}{(2 \pi)^{3}} \mathrm{e}^{-\mathrm{i} p^{0}\left(x^{0}-y^{0}\right)+\mathrm{i} p^{2}\left(x^{2}-y^{2}\right)+\mathrm{i} p^{3}\left(x^{3}-y^{3}\right)} \\
& \times \sqrt{\frac{e B}{\pi}} \mathrm{e}^{-\frac{1}{2} e B\left[\left(x^{1}-\frac{p^{2}}{e B}\right)^{2}+\left(y^{1}-\frac{p^{2}}{e B}\right)^{2}\right]} \frac{\mathrm{i} P_{0}}{p_{\bar{\mu}} \gamma^{\bar{\mu}}-m+\mathrm{i} \epsilon} .
\end{aligned}
$$

with the spin projection matrix; $P_{0}=\operatorname{diag}(1,0,1,0)$. The Landau zero-mode exists for one spin state only and $P_{0}$ is nothing but the projection matrix onto this allowed spin state. In the above the index $\bar{\mu}$ refers to only the 0 th and 3rd components, namely, $\bar{p} \cdot \gamma=p_{\bar{\mu}} \gamma^{\bar{\mu}}=p^{0} \gamma^{0}-p^{3} \gamma^{3}$. One might have thought that $p^{2} / e B$ on the exponential could be dropped in the strong $B$ limit. We must keep this term, however, because the integration variable $p^{2}$ could take any large number. In fact, as explained below, the momentum conservation is guaranteed by the $p^{2}$-integration with this $p^{2} / e B$ term left.

We are now ready to go into concrete calculation of the polarization tensor [1], which has been discussed repeatedly in various contexts such as the magnetic catalysis [12], the chiral magnetic effect [13], and so on. In this sense the computation and the result of the polarization tensor have been already known. Our emphasis in this work is thus put in the physical interpretaion deduced from the energy and momentum dependence in the polarization tensor.

The one-loop contribution is, from the di-

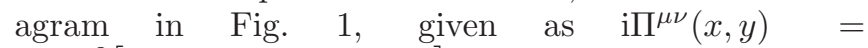
$-(\mathrm{i} e)^{2}\left[\gamma^{\mu} S_{0}(x, y) \gamma^{\nu} S_{0}(y, x)\right]$. Here we consider the polarization for the photon, and if it is for the gluon the coupling constant should be $g$ and the trace in color and flavor space is necessary. We shall work in momentum space to calculate,

$$
\mathrm{i} \Pi^{\mu \nu}(k, q)=\int \mathrm{d}^{4} x \mathrm{~d}^{4} y \mathrm{e}^{\mathrm{i} q \cdot x+\mathrm{i} k \cdot y} \mathrm{i} \Pi^{\mu \nu}(x, y) .
$$

Here $q$ and $k$ are incoming momenta from the external legs attached to $x$ and $y$, respectively. Usually, without external magnetic field, $\Pi^{\mu \nu}(x, y)$ is a function of $x-y$ due to the translational invariance, which can be Fourier decomposed into $\exp [-\mathrm{i} p \cdot(x-y)]$ and $\exp \left[-\mathrm{i} p^{\prime} \cdot(y-x)\right]$ with loop momenta $p$ and $p^{\prime}$ (see Fig. 11), so that $x$ and $y$ integrations yield $(2 \pi)^{4} \delta^{(4)}\left(q-p+p^{\prime}\right)$ and $(2 \pi)^{4} \delta^{(4)}(k-$ $\left.p^{\prime}+p\right)$. Then $p^{\prime}$ is fixed as $p-q$ from the former constraint and the latter becomes $(2 \pi)^{4} \delta^{(4)}(k+q)$, meaning that the outgoing momentum $-k$ is exactly balanced with the incoming momentum $q$.

In the presence of the magnetic field, however, the realization of the momentum conservation is non-trivial because the translational invariance is apparently broken (not broken in fact) by the inhomogeneous vector potential $A^{2}=B x$ and $\Pi^{\mu \nu}(x, y)$ is no longer a function of $x-y$ alone. Although the final result is known (see Eq. (117) in Ref. [12]), it would be instructive to make an explicit confirmation in the following.

For $B \neq 0$ the integrations with respect to $x^{0}, y^{0}$, $x^{2}, y^{2}, x^{3}, y^{3}$ are just the standard procedures leading to the delta-function constraints which lead to $p^{0}=p^{0}-q^{0}$, $p^{\prime 2}=p^{2}-q^{2}, p^{\prime 3}=p^{3}-q^{3}$, and $k^{0}+q^{0}=0, k^{2}+q^{2}=0$, $k^{3}+q^{3}=0$. We can further carry out the $x^{1}$ and $y^{1}$ integrations to have

$$
\begin{aligned}
& \int \mathrm{d} x^{1} \sqrt{\frac{e B}{\pi}} \mathrm{e}^{-\mathrm{i} q^{1} x^{1}} \mathrm{e}^{-\frac{1}{2} e B\left[\left(x^{1}-\frac{p^{2}}{e B}\right)^{2}+\left(x^{1}-\frac{p^{\prime 2}}{e B}\right)^{2}\right]} \\
& =\exp \left\{-\frac{1}{4 e B}\left[\left(q^{1}\right)^{2}+\left(q^{2}\right)^{2}+4 \mathrm{i} q^{1} p^{2}-2 \mathrm{i} q^{1} q^{2}\right]\right\}
\end{aligned}
$$

for the $x^{1}$-integration. Together with the contribution from the $y^{1}$-integration we find,

$$
\exp \left\{-\frac{1}{4 e B}\left[\left(q^{1}\right)^{2}+\left(k^{1}\right)^{2}+2\left(q^{2}\right)^{2}+2 \mathrm{i}\left(q^{1}+k^{1}\right)\left(2 p^{2}-q^{2}\right)\right]\right\} \text {. }
$$

Then, since the fermion propagator does not depend on $p^{2}$, it is straightforward to perform the $p^{2}$-integration and we get $\int \frac{\mathrm{d} p^{2}}{2 \pi} \exp \left[-\frac{\mathrm{i}}{e B}\left(q^{1}+k^{1}\right) p^{2}\right]=2 \pi \delta\left(q^{1}+k^{1}\right) \cdot \frac{e B}{2 \pi}$. This is how we can obtain the momentum conservation and the overall factor $e B / 2 \pi$ that is exactly the Landau degeneracy factor.

After all, in the dimensional regularization, the polarization tensor can be expressed as

$$
\Pi^{\mu \nu}(k, q)=(2 \pi)^{4} \delta^{(4)}(k+q) \mathrm{e}^{-\frac{1}{2 e B}\left[\left(q^{1}\right)^{2}+\left(q^{2}\right)^{2}\right]} \Pi^{\mu \nu}(q)
$$

with

$$
\Pi^{\mu \nu}(q)=\left(g^{\bar{\mu} \bar{\nu}}-\frac{q^{\bar{\mu}} q^{\bar{\nu}}}{\bar{q}^{2}}\right) \frac{e^{2}|e B|}{2 \pi^{2}} I(\bar{q} / m),
$$

where $\Pi^{1 \nu}=\Pi^{2 \nu}=\Pi^{\mu 1}=\Pi^{\mu 2}=0$, which is concluded from $P_{0} \gamma^{1} P_{0}=P_{0} \gamma^{2} P_{0}=0$. In the final expression we have defined a function $I(\bar{q} / m)$ by

$$
I(x)=\int_{0}^{1} \mathrm{~d} y \frac{y(1-y)}{y(1-y)-x^{-2}}=1-\frac{4 \sin ^{-1}(x / 2)}{x \sqrt{4-x^{2}}},
$$




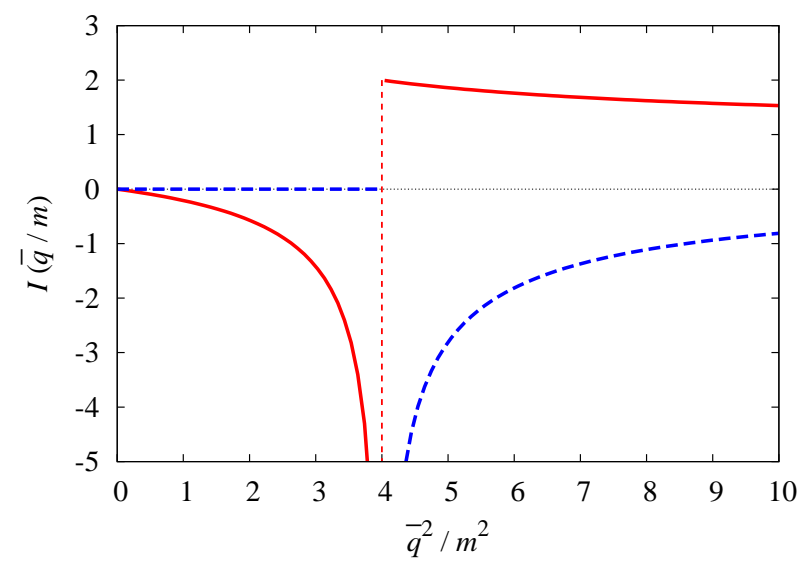

FIG. 2. $I(\bar{q} / m)$ as a function of $\bar{q}^{2} / m^{2}$. The solid and the dashed lines represent the real-part and the imaginary-part, respectively. The mass threshold for particle-anti-particle decay is located at $\bar{q}^{2} / m^{2}=4$.

for $x<2$, namely, $|\bar{q}|<2 m$. This should be expressed as

$$
I(x)=1-\frac{2}{x \sqrt{x^{2}-4}}\left(\ln \left|\frac{x-\sqrt{x^{2}-4}}{x+\sqrt{x^{2}-4}}\right|+\mathrm{i} \pi\right),
$$

for $x>2$, namely, $|\bar{q}|>2 m$ with $q^{0}>0$ assumed. [Because we are considering the retarded self-energy, the sign of the imaginary part changes if $q^{0}<0$.] An imaginary part appears in this case because the gauge particle can decay into a fermionic particle and an anti-particle above the mass threshold.

We make a plot in Fig. 2 to show the behavior of the function $I(\bar{q} / m)$. We can clearly notice that the mass threshold for particle-anti-particle decay is located at $|\bar{q}|=2 m$ where the real-part diverges if it is approached from the smaller- $\bar{q}$ side, while the imaginary-part diverges if approached from the larger $\bar{q}$ side. In the polarization tensor in $(3+1)$ dimensions there would be no such singularity at the mass threshold because of the phase-space factor that compensates for the divergence.

In the definition (9) we can readily take the limit of $m \rightarrow 0$, i.e. $x \rightarrow \infty$ to obtain $I(\infty)=1$. Therefore, in this limit, the polarization simply becomes $\Pi^{\mu \nu}(q)=\left(g^{\bar{\mu} \bar{\nu}}-q^{\bar{\mu}} q^{\bar{\nu}} / \bar{q}^{2}\right) e^{2}|e B| / 2 \pi^{2}$. This result has an interpretation as the (1+1)-dimensional self-energy $\left(g^{\bar{\mu} \bar{\nu}}-q^{\bar{\mu}} q^{\bar{\nu}} / \bar{q}^{2}\right) m_{\gamma}^{2}$ multiplied by the Landau-level degeneracy $|e B| / 2 \pi$, where $m_{\gamma}^{2}=e^{2} / \pi$ is the "photon mass" in the massless Schwinger model [14]. Since the massless Schwinger model is exactly solvable and the dimensional reduction is complete as we checked above, this result at $m=0$ is presumably exact.

Here we shall make a rather technical remark about the momentum integration. The naïve momentum integration for the one-loop polarization in $(1+1)$ dimensions is UV finite. Nevertheless, the final results could depend on a choice of the regularization procedure. To see this, let us take an example of $\Pi^{00}\left(q^{0}=0,|\boldsymbol{q}| \rightarrow 0\right)$ in the $m=0$ limit. From Eq. (8) we already know that the correct answer should be $e^{2}|e B| / 2 \pi^{2}$ in this limit. If we perform the $p^{0}$-integration first [15], however, we can easily prove,

$$
\lim _{q \rightarrow 0} \int \frac{\mathrm{d} p^{0}}{2 \pi} \frac{2\left(p^{0}\right)^{2}-\bar{p} \cdot(\bar{p}-\bar{q})}{\bar{p}^{2}(\bar{p}-\bar{q})^{2}}=0 .
$$

Then, one may conclude that $\Pi^{00}\left(q^{0}=0,|\bar{q}| \rightarrow 0\right)=0$ which is obviously different from the correct answer in the dimensional regularization. One can argue the computation of $\Pi^{33}\left(q^{0}=0,|\bar{q}| \rightarrow 0\right)$ in the same way to find a discrepancy again. Such subtlety originates from the fact that the limit of $|\bar{q}| \rightarrow 0$ is not commutative to the limit of $m^{2} \rightarrow 0$ as is noticeable from Fig. 2. In other words the physical properties in the region with $|\bar{q}|<2 m$ are not accessible at all by the knowledge from the massless Schwinger model. We pay our attention to this subtlety since it is common to process the $p^{0}$-integration first in finite- $T$ calculations. The gauge invariant results (8) implies that the electric-current susceptibility at vanishing momenta is zero, while the naïve estimates are not [15]. Moreover, interestingly, a statement similar to Eq. (11) is correct for the matter part involving the Fermi-Dirac distribution function at finite $T$. We can explicitly make sure that the matter part with $m=0, q^{0}=0$, and $|\boldsymbol{q}| \rightarrow 0$ is vanishing just like Eq. (11). The finite- $T$ extension will be reported elsewhere.

Now we can write the inverse propagator of gauge particles as

$$
\begin{aligned}
-\mathrm{i} D_{R}^{-1 \mu \nu}(q)=g^{\mu \nu} q^{2} & -q^{\mu} q^{\nu}-\Pi^{\mu \nu}(q) \\
& +(\text { gauge fixing terms }) .
\end{aligned}
$$

We note that we neglect the exponential factor $\mathrm{e}^{-\frac{1}{2 e B}\left[\left(q^{1}\right)^{2}+\left(q^{2}\right)^{2}\right]}$ assuming that the transverse external momenta are sufficiently smaller than $e B$, for which the LLL approximation can be justified. Let us clarify what collective excitations are contained in the above propagator. For this purpose we should fix the gauge first and locate the pole(s) of $D_{R}\left(\omega_{q}, \boldsymbol{q}\right)$ as a function of $\omega_{q}$ for a given $\boldsymbol{q}$. Instead we can locate the pole position by solving the equation, $\operatorname{det}\left[D_{R}^{-1}\left(\omega_{q}, \boldsymbol{q}\right)\right]=0$.

Here we adopt the temporal axial gauge $A^{0}=0$ so that the physical degrees of freedom is intuitively manifest. Then, $D_{R}^{-1}$ is a $3 \times 3$ matrix in the Lorentz index and we can show that

$$
\begin{aligned}
\operatorname{det}\left[D_{R}^{-1}\right] & =\operatorname{det}\left[-q^{2} \delta^{i j}-q^{i} q^{j}+\delta^{i 3} \delta^{j 3} \frac{\left(q^{0}\right)^{2}}{\bar{q}^{2}} \mathcal{M}^{2}(q)\right] \\
& =-\left(q^{0}\right)^{2} q^{2}\left[q^{2}-\mathcal{M}^{2}(q)\right],
\end{aligned}
$$

where $\mathcal{M}^{2}=\left(e^{2}|e B| / 2 \pi^{2}\right) I(\bar{q} / m)$. The first factor $\left(q^{0}\right)^{2}$ is from the longitudinal gauge field and this unphysical contribution is exactly cancelled by the Faddeev-Popov determinant associated with the $A^{0}=0$ gauge. The next factor represents one of two transverse gauge fields whose dispersion relation is intact. The final factor is 


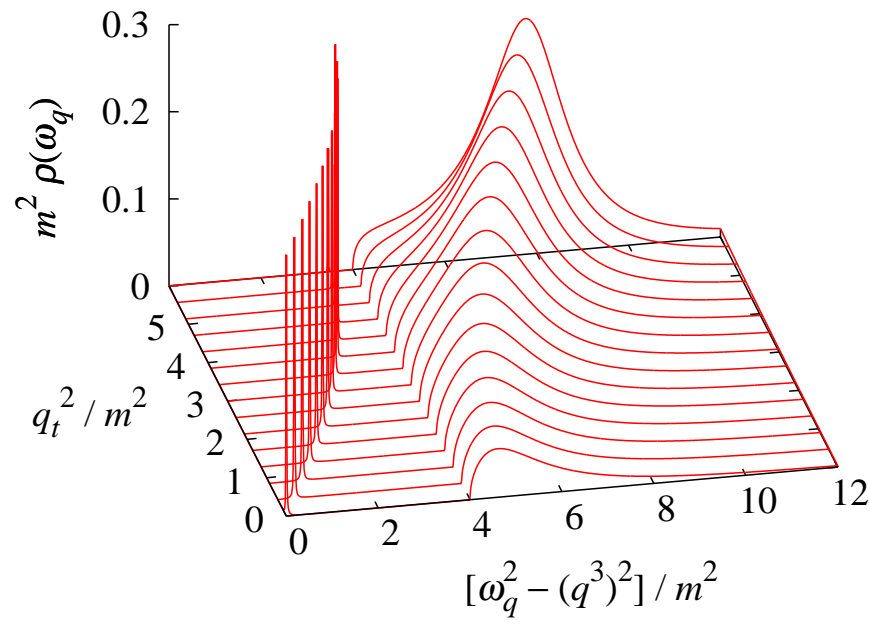

FIG. 3. Spectral function which is made dimensionless by $m^{2}$. We choose $e^{2}|e B| /\left(2 \pi m^{2}\right)=1$.

the most interesting; one of two transverse gauge fields is influenced by the screening effect by $\mathcal{M}^{2}(q)$.

Solving $q^{2}-\mathcal{M}^{2}(q)=0$, we can figure out the energy dispersion relation $\omega_{q}(\boldsymbol{q})$ of the collective excitation in the same way as reading the plasmon dispersion at finite temperature. For intuitive understanding we do not show the pole position but discuss the spectral function which contains the information of both the energy dispersion relation and the decay width. Instead of defining the spectral function from $D_{R}(q)$ directly, we define $\rho\left(\omega_{q}\right)$ for the transverse mode affected by $\mathcal{M}^{2}(q)$ as if it were a scalar field, that is,

$$
\rho\left(\omega_{q}\right)=-\frac{1}{\pi} \frac{\operatorname{Im} \mathcal{M}^{2}(q)}{\left[q^{2}-\operatorname{Re} \mathcal{M}^{2}(q)\right]^{2}+\left[\operatorname{Im} \mathcal{M}^{2}(q)\right]^{2}} .
$$

Figure 3 is the numerical result when we set $e^{2}|e B| /\left(2 \pi m^{2}\right)=1$. Actually changing $e^{2}|e B| / 2 \pi m^{2}$ makes no qualitative difference. We can see that there are two separate branches below and above the mass threshold. In what follows we discuss them in order.

Let us look at the branch above the threshold, i.e. $|\bar{q}|=\sqrt{\omega_{q}^{2}-\left(q^{3}\right)^{2}}>2 m$. In this case the collective excitation (photon) is attenuated because it can decay into a particle and an anti-particle. The width is divergently large near the threshold, which is already clear from the dashed line in Fig. 2] As the transverse momentum $q_{t}^{2}=\left(q^{1}\right)^{2}+\left(q^{2}\right)^{2}$ increases the width becomes narrower and narrower. In the limit of $q_{t}^{2} \gg m^{2}$, eventually, the dispersion is reduced to that of massive photon with its mass given by $e^{2}|e B| / 2 \pi^{2}$. This asymptotic situation is, as already mentioned, the limit toward the massless Schwinger model.

We can notice that the spectral height grows larger as $q_{t}^{2}$ increases. The spectral sum rule should be preserved with the contribution from another branch below the mass threshold. As noticed from Fig. 3 this collective mode below the threshold lies in the space-like region $\left(\omega_{q}^{2}<|\boldsymbol{q}|^{2}\right)$ without attenuation, which looks like a sound mode. Such an observation of the sound-like mode has been also reported recently in the strong-coupling regime in the AdS/CFT analysis [16]. Since we are working diagramatically and the physical contents are transparent, we can give a clear interpretation for this collective excitation in our perturbative approach; we propose that this should be an analogue of the zero sound in the Fermi liquid theory [17].

Because of the dimensional reduction, on the one hand, physical properties of the system in a strong magnetic field looks like $(1+1)$ dimensional. At finite density, on the other hand, physical properties are dominated by the interactions near the Fermi surface, so that the dynamics is effectively $(1+1)$ dimensional too. [Since the momentum in a certain direction is as large as the chemical potential, the momenta in other directions perpendicular to the motion are negligible and the system is locally $(1+1)$ dimensional if the Fermi momentum is large enough.] In condensed matter physics the zero sound appears in Fermi liquid even at zero temperature as a result of the particle-hole excitation in pseudo $(1+1)$ dimensions where the polarization has a logarithmic singularity similar to our Fig. 2 In the present case, the magnetic field instead of the Fermi surface causes the pseudo $(1+1)$ dimensionality, and the particle-anti-particle excitation instead of the particle-hole excitation constitute sound-like collectivity in the vector channel.

Usually the zero sound is a longitudinal sound mode associated with the density fluctuation which appears only when the Landau parameter $F_{0}$ in the fermionic interaction is repulsive. In relativistic matter the zero sound emerges as a gapless branch of the vector meson [18]. It is remarkable that in our case the zero-sound-like mode lies in the transverse degrees of freedom, so that it is indistinguishable from the massless photon even though the microscopic content is totally different.

In this work we focus on the $B$-induced screening effects on massless gauge particles only. It would be very interesting to think about the screening effects on massive vector mesons in the same way as in Ref. [18]. We would then anticipate that a massless collective mode should exist in the space-like region as discussed in Ref. [18], which is the complete counterpart of the zero sound, which we name the "magnetic zero sound" here. Recently it was pointed out that the $\rho$-meson superconductor could be possible in the vacuum with a superstrong $B$ field [19]. It is an important question how this electric superconductivity should or should not be modified by the screening effects and particularly by the existence of the magnetic zero sound.

In summary, in the LLL approximation, we found that one of transverse gauge particles is screened by the magnetic field, which leads to two branches of the collective excitations. One has a modified dispersion relation with a mass and a width whose behavior is reminiscent of the plasmon at finite temperature. The other looks like the zero sound at finite density, which can be naturally un- 
derstood from the pseudo $(1+1)$ dimensional nature under a strong magnetic field. These modes are relevant to the situation with $|e B| \gg m^{2} \sim|\boldsymbol{q}|^{2}$ and convey detailed information of the birefringence in the vacuum (not medium) with a magnetic field background. Because a huge $|e B|$ is expected in the heavy-ion collision, we may have a chance to probe these collective modes indeed. If we replace the overall coupling constant $e^{2}$ in $\mathcal{M}^{2}$ by $g^{2}$ for quark loops, it would enhance the chance for observation and also we can discuss the physics implication of our results to the QCD phenomena such as the chiral

[1] For reviews, see; G. V. Dunne, In *Shifman, M. (ed.) et al.: From fields to strings, vol. $1 * 445-522$; G. A. Mourou, T. Tajima, S. V. Bulanov, Rev. Mod. Phys. 78, 309-371 (2006).

[2] R. Schutzhold, H. Gies and G. Dunne, Phys. Rev. Lett. 101, 130404 (2008); F. Hebenstreit, R. Alkofer, G. V. Dunne and H. Gies, Phys. Rev. Lett. 102, 150404 (2009); S. S. Bulanov, V. D. Mur, N. B. Narozhny, J. Nees and V. S. Popov, Phys. Rev. Lett. 104, 220404 (2010).

[3] V. P. Gusynin, V. A. Miransky, I. A. Shovkovy, Phys. Rev. Lett. 73, 3499-3502 (1994); Phys. Rev. D52, 47184735 (1995).

[4] D. E. Kharzeev, L. D. McLerran, H. J. Warringa, Nucl. Phys. A803, 227-253 (2008).

[5] V. Skokov, A. Y. .Illarionov, V. Toneev, Int. J. Mod. Phys. A24, 5925-5932 (2009).

[6] M. A. Metlitski and A. R. Zhitnitsky, Phys. Rev. D 72, 045011 (2005); G. M. Newman and D. T. Son, Phys. Rev. D 73, 045006 (2006); D. Kharzeev and A. Zhitnitsky, Nucl. Phys. A 797, 67 (2007); K. Fukushima, D. E. Kharzeev, H. J. Warringa, Phys. Rev. D78, 074033 (2008); D. E. Kharzeev, D. T. Son, Phys. Rev. Lett. 106, 062301 (2011); Y. Burnier, D. E. Kharzeev, J. Liao, H. U. Yee, arXiv:1103.1307 [hep-ph].

[7] P. V. Buividovich, M. N. Chernodub, E. V. Luschevskaya, M. I. Polikarpov, Phys. Rev. D80, 054503 (2009); P. V. Buividovich, M. N. Chernodub, E. V. Luschevskaya, M. I. Polikarpov, Phys. Rev. D81, 036007 (2010); P. V. Buividovich, M. N. Chernodub, D. E. Kharzeev, T. Kalaydzhyan, E. V. Luschevskaya, M. I. Polikarpov, Phys. Rev. Lett. 105, 132001 (2010); magnetic effect. Using the NJL-type models we can also discuss the realization of the magnetic zero sound in the excitation spectra of vector mesons. These applications are interesting future problems.

The author thanks Wolfram Weise for useful comments and his kind hospitality at TUM where this work was complete. He also thanks Dmitri Kharzeev and Harmen Warringa for discussions that inspired him toward this work. He is grateful to Maxim Chernodub for valuable comments. He was supported by Grant-in-Aid for Young Scientists B (No. 20740134).
M. Abramczyk, T. Blum, G. Petropoulos, R. Zhou, PoS LAT2009, 181 (2009).

[8] A. J. Mizher, M. N. Chernodub, E. S. Fraga, Phys. Rev. D82, 105016 (2010); K. Fukushima, M. Ruggieri, R. Gatto, Phys. Rev. D81, 114031 (2010); R. Gatto, M. Ruggieri, Phys. Rev. D83, 034016 (2011).

[9] E. J. Ferrer, V. de la Incera, C. Manuel, Phys. Rev. Lett. 95, 152002 (2005); Nucl. Phys. B747, 88-112 (2006); E. J. Ferrer, V. de la Incera, Phys. Rev. Lett. 97, 122301 (2006); J. L. Noronha, I. A. Shovkovy, Phys. Rev. D76, 105030 (2007); K. Fukushima, H. J. Warringa, Phys. Rev. Lett. 100, 032007 (2008).

[10] V. I. Ritus, Annals Phys. 69 (1972) 555.

[11] U. H. Danielsson, D. Grasso, Phys. Rev. D52, 2533-2542 (1995); V. Zeitlin, J. Exp. Theor. Phys. 82, 79-84 (1996); R. Sharma, S. Reddy, Phys. Rev. C83, 025803 (2011).

[12] V. P. Gusynin, V. A. Miransky and I. A. Shovkovy, Nucl. Phys. B 462, 249 (1996).

[13] D. E. Kharzeev, H. J. Warringa, Phys. Rev. D80, 034028 (2009).

[14] J. S. Schwinger, Phys. Rev. 128, 2425-2429 (1962).

[15] K. Fukushima, D. E. Kharzeev, H. J. Warringa, Nucl. Phys. A836, 311-336 (2010); K. Fukushima, M. Ruggieri, Phys. Rev. D82, 054001 (2010).

[16] D. E. Kharzeev, H. -U. Yee, arXiv:1012.6026 [hep-th].

[17] For a textbook; see for example, A. A. Abrikosov, L. P. Gorkov, and I. E. Dzyloshinski, Methods of Quantum Field Theory in Statistical Physics (Dover, New York, 1975).

[18] S. A. Chin, Annals Phys. 108, 301 (1977).

[19] M. N. Chernodub, Phys. Rev. D82, 085011 (2010); arXiv:1011.2658 [hep-ph]. 